\title{
The Role of Personalization and Multiple EEG and Sound Features Selection in Real Time Sonification for Neurofeedback
}

\author{
S. Mealla ${ }^{1}$, A. Oliveira ${ }^{1}$, X. Marimon ${ }^{2}$, T. Steffert $^{3}$, S. Jordà̀ $^{1}$ and A. Väljamäe ${ }^{4,5}$ \\ ${ }^{1}$ Music Technology Group (MTG), Department of Information and Communication Technologies (DTIC), Universitat \\ Pompeu Fabra, Barcelona \\ ${ }^{2}$ BarcelonaTECH, LASSIE Lab, Automatic Control Department, Universitat Politecnica de Catalunya, Barcelona \\ ${ }^{3}$ Centre for Research in Computing, The Open University, Milton Keynes, UK \\ ${ }^{4}$ Department of Higher Nervous Activity, St. Petersburg State University, St. Petersburg, Russia \\ ${ }^{5}$ Department of Behavioural Sciences and Learning, Linköping University, Linköping, Sweden \\ \{sebastian.mealla,sergi.jorda\}@upf.edu, \{aluiziob.oliveira,xavier.marimon.serra\}@gmail.com, tony@qeeg.co.uk, \\ aleksander.valjmae@liu.se
}

Keywords: Sonification, EEG, alpha/theta neurofeedback, Physiological Computing, Pure Data, Sound, Real Time

Abstract: The field of physiology-based interaction and monitoring is developing at a fast pace. Emerging applications like fatigue monitoring often use sound to convey complex dynamics of biological signals and to provide an alternative, non-visual information channel. Most Physiology-to-Sound mappings in such auditory displays do not allow customization by the end-users. We designed a new sonification system that can be used for extracting, processing and displaying Electroencephalography data (EEG) with different sonification strategies. The system was validated with four user groups performing alpha/theta neurofeedback training (a/t) for relaxation that varied in feedback personalization (Personalized/Fixed) and a number of sonified EEG features (Single/Multiple). The groups with personalized feedback performed significantly better in their training than fixed mappings groups, as shown by both subjective ratings and physiological indices. Additionally, the higher number of sonified EEG features resulted in deeper relaxation than when training with single feature feedback. Our results demonstrate the importance of adaptation and personaliziation of EEG sonification according to particular applications, in our case, to a/t neurofeedback. Our experimental approach shows how user performance can be used for validating different sonification strategies.

\section{INTRODUCTION}

Recent advances in human physiological sensors and signal processing techniques have fostered the development of computer systems for online control, training and monitoring of various cognitive and affective states (Allanson and Fairclough, 2004). In addition, an increasing number of consumer ofthe-shelf devices and open source platforms transform physiology-based interaction into an important part of the Human Computer Interaction (HCI) field. Systems like the Emotiv EPOC (Emotiv, 2013), or Starlab's Enobio (Neuroelectrics, 2013) now allow a low cost and wearable electroencephalography (EEG) sensing in out-of-the-lab, real life conditions, increasing "external validity" of conducted research.

Sonification techniques have been widely used to display EEG activity and are often an integral part of physiological computing. Besides being ide- ally suited for encoding complex dynamic structures due to auditory perception characteristics (Guttman et al., 2005), sound provides a complementary sensory channel complementing visual displays. Hence, EEG sonification is a growing field of auditory displays that embrace several application domains including HCI, Brain-Computer Interfaces (BCI), EEGbased music, neurofeedback (NF) and EEG monitoring (see (Väljamäe et al., 2013) for a review).

The selection of functional EEG-to-Sound transformations is a difficult task involving perceptual and aesthetical trade-offs depending on their application and goals (Hermann et al., 2002). On the one hand, simple EEG-to-sound mappings allow direct perception of changes in the EEG, producing almost reversible signals, e.g., EEG time compression with the purpose of pre-screening specific brain activity events (Khamis et al., 2012). However, such sonifications tend to be unnatural and not well suited for hearing 
out multiple EEG events. On the other hand, more indirect transformations provide more naturalistic, rich and perceptually pleasant sonifications. The trade-off here is an arbitrary mapping, as, for example, with EEG driven musical content in (Mullen et al., 2011) that hides original physiological features from listeners. In this context, end-users impression of the designed sonifications becomes a crucial factor for the application success.

Regrettably, most of the available EEG sonifications are working with a constant and predefined number of EEG features, and also apply fixed, predefined EEG-to-Sound mappings that cannot be changed by end users. De Campo and colleagues conducted one of the few studies addressing the personalization of EEG sonification, i.e. adjustment of display parameters by end users (De Campo et al., 2007). Tests were done with medical specialists performing evaluation of EEG data containing epileptic events and seizures and showed the importance of sonification parameters customization.

To study both varying content (richness) and personalization aspects of auditory display for physiological data, we created a real-time sonification system that accounts for a flexible multi-parametric EEG signal transformation into sound, also allowing users to personalize EEG-to-Sound mappings in real time. We tested our EEG sonification system in an empirical study based on well-established alpha/theta $(\mathrm{a} / \mathrm{t}$ ) neurofeedback training paradigm (see (Gruzelier, 2009) for a review). During this training, users try to relax with their eyes being closed. The most important moment is the so-called theta/alpha "crossover" when alpha activity slowly subsides accompanying sleep onset and theta activity becomes more dominant (Egner et al., 2002). The increase of $t / a$ power ratio with eyes closed is a well known accompaniment of states of deep relaxation such as stage 1 of sleep and meditation (Gruzelier, 2009).

As the a/t NF training is typically based on auditory feedback, it is a good candidate to validate the effectiveness of personalization and sonification richness of our auditory display. We used our sonification system to present a pleasant soundscape contingent to the production of theta, alpha, and/or $t / a$ ratio. Specifically, we hypothesized that a/t NF training would have:

- Hypothesis 1 - More impact for participants that are using personalized sonification mappings than using pre-defined, fixed sound mappings;

- Hypothesis 2- More impact for participants using multiple EEG feature sonification compared to one only based on $t / a$ ratio;

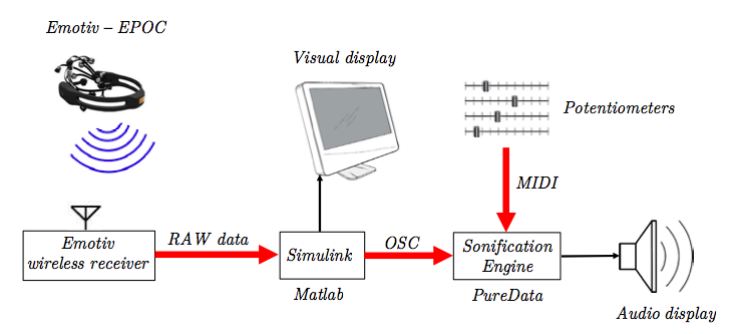

Figure 1: Components and data streams of the sonification system: EEG signal acquisition, signal extraction and processing, and sonification engine.

\section{METHODS}

\subsection{Equipment and Instrumentation}

The system is composed of three main blocks: (1) EEG signal acquisition, (2) signal extraction and processing, and (3) the sonification engine. It allows designers to build customized and versatile mapping definitions. Figure 1 shows the architecture of the sonification system.

\subsubsection{EEG signal acquisition}

EEG data is acquired using an Emotiv EPOC, a wireless, non-invasive 14-channel EEG headset (Emotiv, 2013). The EPOC is one of the leaders in low-cost consumer EEG sensing devices. Although it can not be reliably used for most BCI applications since its signal-to-noise levels are lower than in medical devices (Duvinage et al., 2013), it has been used successfully for user states monitoring (Rodríguez et al., 2013). The EPOC's electrodes require a saline solution to improve conductivity, and their placement is related to the 10-20 system. EPOCs data is sampled at $128 \mathrm{~Hz}$ and low- and high-pass filtered internally at $85 \mathrm{~Hz}$ and $0.16 \mathrm{~Hz}$ respectively. This semi-raw data is accessed through the EPOC SDK (Emotiv, 2013) and then accessed by Matlab/Simulink model.

\subsubsection{Signals extractions and processing}

We designed a toolbox for Matlab/Simulink to process EPOCs EEG data, both online and offline, and to feed the sonification engine. A number of processing blocks described below allow extracting EEG features in a modular way, adapting to different neurofeedback or monitoring strategies.

- Emotiv2Simulink, based on a Mex S-function and on the drivers provided by the manufacturer, it allows the access to the raw data of the EPOC.

- BP filters, Butterworth IIR discrete band-pass (BP) filters that can be tuned to any given custom 
frequency.

- Envelope, is a block that squares the input signal and then applies a FIR low-pass filter and downsampling to estimate its envelope.

- MaxMin, is a block based on a configurable window of size $L$; it estimates the maximum, minimum and median value of a signal in the temporal and frequency domain.

- Hjorth Descriptors, calculate the Hjorth parameters for activity, mobility, and complexity (Liang et al., 2013)(Hjorth, 1970).

- OSCSend, allows direct communication via UDP with virtually any modern real-time sound synthesis environment through the Open Sound Control (OSC) protocol(Wright, 2005).

\subsubsection{Sonification engine}

A versatile sonification engine was developed using PureData (Pd) (Puckette et al., 1996). The engine has been built following a modular approach that allows sonification designers to choose among different EEG features (processed in the Simulink toolbox) and their number, and to define a certain EEG-to-Sound mapping. Although the system has been tested with only 3 EEG features feeding 5 sound modules in parallel, there are no restrictions on the number of modules to be displayed, other than hardware limitations (i.e. processing power). Multiple EEG streams also can feed a single sound module. Importantly, the engine also allows further adjustment by end users via MIDI controllers.

Below we provide a description of the different modules of the sonification engine. For flexibility and easiness, all inputs of the sonification engine modules are normalized between 0 and 100 .

Pedal module. A fixed pitch tonic sound is initialized on loading (D2, MIDI note $n^{\circ} 38$ ). A tremolo effect is applied and phase shifted to each of the even harmonics, giving a slowly moving chorus-like timbre to the drone. Inputs for this module are able to control tremolo, panning and gain.

Melody module. The melody is constructed using a major scale stemming from five semitones (one fourth) bellow the central tonic to sixteen semitones above it (major third). The slope of the incoming signal controls note triggering speed and pitch, whereas the volume is controlled by a linear function of the input signal.

Rewarding module. This module triggers short duration nature sounds (birds, owls, and crickets) when the input signal goes over a predefined threshold value. Therefore, every time the input signal is over the threshold set, the user is rewarded with a more varying yet still relaxing soundscape. A dynamic amplitude panning allows use of spatial audio.

Wind model. Based on a procedural audio, this module use a series of white noise generators to generate a wind soundscape. The use of procedural audio (in contrast to a sample-based approach) gives a complete control over sound parameters (e.g., wind speed). Therefore, the perceptual quality of the windy scene dynamically changes according to mapped EEG features.

Rain model. Based on a sample-based approach, this module preserves soundscape fidelity using dynamic cross fading between different rain excerpts. It also allows a comparison with the procedural audio approach of the Wind Module. Input signal modulates the amount of excerpts in the resulting composite Rain density, which varies from a light rain to a small thunderstorm.

Graphic User Interface and Personalization. A graphic user interface (GUI) was created to simplify the patching between different sound modules and EEG features. It also contains time and frequency signal monitoring scopes for both input (EEG) and output (sound) data. The GUI also displays tools for users' personalization via MIDI controller, allowing them to adjust their mapping "on the fly" while listening to the sonification results in real-time.

Users are able to personalize, i.e. adjust, the mappings as follows. One EEG feature is associated to one sound element (e.g. alpha relative power is mapped to Rain density). The user is then allowed to change the mapping in a continuous scale from -1 to +1 . If the potentiometer is dragged to -1 , the mapping is inversed, i.e. a greater value of the data feature yields a smaller value for the sound parameter (more alpha would lead to less rain). If it is positioned at 1 , the mapping is positive (more alpha yields more rain). In the middle (zero), the sound is fixed and not influenced by the data (a fixed amount of rain equivalent to $50 \%$ of its maximum value, independently of the alpha power).

\subsubsection{Settings used in the experiment}

Figure 2 shows the system configuration for our evaluation study.

The EEG toolbox has been configured to extract three main EEG features, according to the a/t NF protocol (Gruzelier, 2009). In accordance with previous NF studies (Egner et al., 2002), alpha relative power $(8-13 \mathrm{~Hz}$ range) is calculated from the occipital area where this type of activity occurs during close-eye conditions (Kropotov, 2010). The second feature, theta relative power $(4-7.5 \mathrm{~Hz}$ range $)$ is cal- 
Table 1: Configuration of the Sound Engine for each group, defined by type of mapping (Personalized or Fixed) and number of EEG features (Single or Multiple). EEG features include $t / a$ ratio, and relative power of Alpha and Theta). "Personalized" stands for adjusted mappings driven by EEG, "Fixed" stands for fixed mapping driven by EEG, and "Constant" stands for sound feature held constant.

\begin{tabular}{ccccc}
\hline \hline Group & Foreground & Rewarding & Wind & Rain \\
\hline F-S & t/a ratio (Fixed) & t/a ratio & Constant & Constant \\
P-S & t/a ratio (Personalized) & t/a ratio & Constant & Constant \\
F-M & t/a ratio (Fixed) & t/a ratio & theta (Fixed) & alpha (Fixed) \\
P-M & t/a ratio (Personalized) & t/a ratio & theta (Personalized) & alpha (Personalized) \\
\hline
\end{tabular}

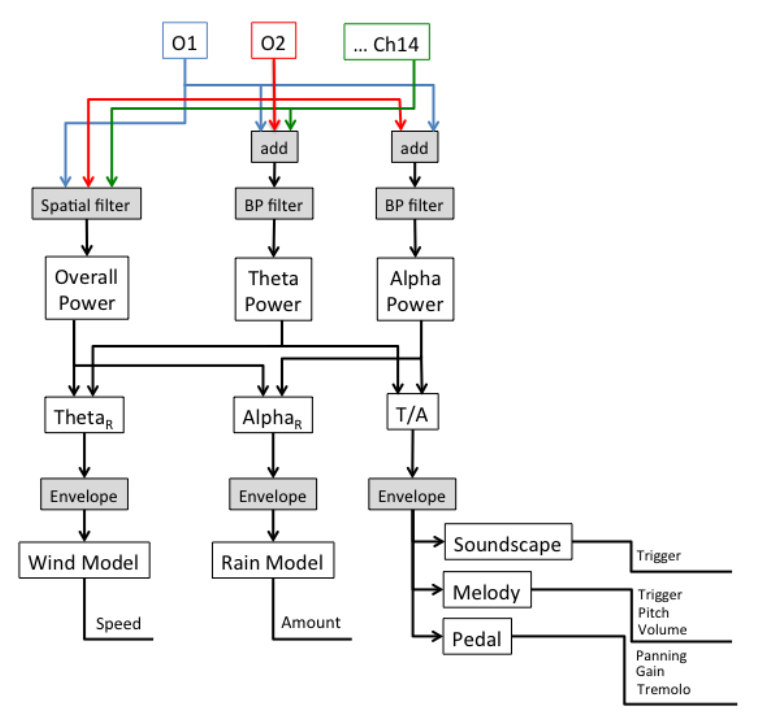

Figure 2: Processing stages of EEG features and corresponding sound mappings

culated from the activity of all 14 channels, as cortical theta rhythms are small and diffuse when picked up by scalp electrode, arising almost entirely from the cerebral cortex (Kropotov, 2010). These two relative powers are obtained by dividing the band power by the overall signal power. In this manner, the output signal is kept within a range of 0 to 1 . Finally, the third feature, $t / a$ power ratio, is estimated as the main measure for the $\mathrm{a} / \mathrm{t} \mathrm{NF}$ procedure. A spatial filtering is applied to give more weight to the occipital channels in the calculation of the envelope of the alpha band and alpha-theta ratio. For the calculation of the envelope of theta, equal weight for all channels is used. The envelope is estimated for all three EEG features using the envelope block based on FIR-based filter (order 35).

The sound engine has been configured as follows (see also table 1):

- Pedal Module: tremolo, panning and gain are linearly mapped to $t / a$ ratio.

- Melody module: note triggering speed and pitch are functions of the $t / a$ ratio where its positive change yield ascending pitches while negative ones leads to descending pitches. Higher rate of change yields bigger jumps in melody (more semitones between two consecutive notes). The note volume is a linear function of $t / a$ ratio.

- Rewarding module: Threshold is set at $50 \%$ of the calibrated maximum value of $t / a$ ratio recorded prior to the main training session.

- Wind model: Theta relative power controls wind speed of the modeled sound object - the higher is the input, the faster the wind will "blow".

- Rain model: Alpha relative power is used to control Rain density.

The experiment was conducted in a room isolated from external noise. Participants were seated on a swivel chair equally distant to four loudspeakers (Roland active Loudspeakers, Model MA15-D, and a M-Audio sound card, FastTrack Pro). Previous research has shown that spatial rendering increases affective impact of sound (Vastfjall, 2003). The lights were dimmed down during the personalization and pre-relaxation sessions (see Procedure section) and turned off during the a/t NF session. A 42 inches screen (not visible to the for participants) was used to monitor the EEG signal quality and levels.

\subsection{Experimental procedure}

Two hypotheses were tested using a betweensubjects design. Thirty one participants, mean age $27.81(S D=5.18), 15$ females, took part in the experiment. The study was conducted in accordance with the Declaration of Helsinki. Participants were randomly distributed among four experimental groups:

- F-S group: Fixed EEG-to-Sound mappings, Single EEG feature sonified $(M=26, S D=2)$;

- P-S group: Personalized mappings, Single EEG feature sonified $(M=27.6, S D=5)$;

- F-M group: Fixed mappings, Multiple EEG features $(M=30, S D=8)$; 
- P-M group: Personalized mappings, Multiple EEG features sonified $(M=27.5, S D=4)$.

All groups listened to soundscapes of a comparable complexity, but these varied in the number of EEG features feeding the system, and in the sonification mappings applied. Table 1 shows the configuration of the sound engine for each group. Each experiment lasted around 50 minutes, according to the following protocol:

- Information and consent form: The participant was explained each stage of the experiment, and the relation between brain activity and the created soundscape; the consent form was signed.

- Initial emotional state self-assessment: Subjective measures of emotional valence and arousal were collected in paper through a 9-point SelfAssessment Manikin (SAM) after (Bradley and Lang, 1994).

- Sensor placement and baseline state recording: The participant was seated in a swivel chair, and the Emotiv EPOC was mounted. The baseline EEG activity was recorded.

- Relaxation induction: The participant was asked to close her eyes and listen to a 5-minute sound of sea waves. EEG activity was recorded, and the thresholds for NF session were calculated by looking at the maxima and minima values.

- Pre-test emotional state self-assessment: The participant filled-in again two SAM scales.

- Mapping adjustment: EEG features were sonified online. The participant was asked to personalize the mappings till reaching the most relaxing sound possible, using a MIDI interface with potentiometers placed in front of the chair. There was no time limit set to define the mappings.

- NF training session: The participant was asked to close her eyes and to turn the chair facing away from the experiment leader. Participants were asked to relax and to listen to the soundscape (different for each group) for 15 minutes. Participants were instructed to raise their hand if feeling uncomfortable or falling asleep.

- Post-test emotional state self-assessment: participants filled-in two SAM scales again.

- Headset removal and debriefing: the EEG headset was removed. Participants were debriefed, thanked and received candies as a small reward.

\subsection{Data Analysis}

For analysis purposes, the raw EEG data with sampling at $128 \mathrm{~Hz}$ was first filtered $(0.5-30 \mathrm{~Hz})$. Using visual inspection and thresholding (over $3 \sigma$ ) data regions with artefacts were marked for removal in subsequent analyses. Closely following the design of the NF training protocol (see section 2.1.4), we analyzed data from O1 and O2 electrodes. Signals were BP filtered to obtain alpha $(8-13 \mathrm{~Hz})$ and theta $(4-7.5 \mathrm{~Hz})$ components. The 15 minute data recordings were split into $10 \mathrm{~s}$ epochs and for each of them relative alpha, relative theta and $t / a$ ratio where calculated. Next, we averaged the means from individual epochs for five $3-\min$ periods (18 epochs each) excluding the epochs marked as containing artifacts. This was done for $\mathrm{O} 1$ and $\mathrm{O} 2$ channels separately. Then we used the values averaged across both channels and used the means from the first and the last $3-\min$ period of experimental procedure for analysis. In other words, we compared maximal changes caused by 15 minute training in relative alpha, relative theta and $t / a$ ratio.

Both subjective (SAM ratings of pre and post assessment of emotional state) and objective measures (EEG features) of two relaxation periods were subjected to 3-way MANOVA. Therefore within-subjects factor was Relaxation periods (1-3 min vs. 12-15 min period), whereas between-subjects factors were Number of sonified EEG features (Single vs. Multiple) and Feedback personalization (Personalized vs. Fixed mapping). Alpha level was fixed at 0.05 for all statistical tests. Greenhouse-Geisser correction was used to correct for unequal variances. For multivariate analysis Wilks' Lambda $\Lambda$ was used as the multivariate criterion. All variables were normally distributed according to the Kolmogorov-Smirnov test.

\section{RESULTS}

In accordance with the relaxing nature of the experimental procedure, the overall MANOVA effect of Relaxation period was significant with $F(5,23)=$ $6.89, p<0.001, \Lambda=0.4, \hat{\eta}_{P}^{2}=0.6$. Split by measures, this effect reached significance for: subjective arousal ratings, $F(1,27)=26.06, p<0.001, \hat{\eta}_{P}^{2}=0.49$; for relative alpha power, $F(1,27)=5.81, p<0.05, \hat{\eta}_{P}^{2}=$ 0.18 ; for relative theta power, $F(1,27)=10.4, p<$ $0.005, \hat{\eta}_{P}^{2}=0.28$; and for $t / a$ ratio, $F(1,27)=$ $5.45, p<0.05, \hat{\eta}_{P}^{2}=0.17$. This shows that participants in all four groups reached a certain level of relaxation as compared with the initial 3 minute period of the experiment. More importantly, the interaction between the Relaxation period and betweengroup factors of Number of sonified EEG features and Feedback personalization also showed significance.

Confirming our Hypothesis 1, the over- 

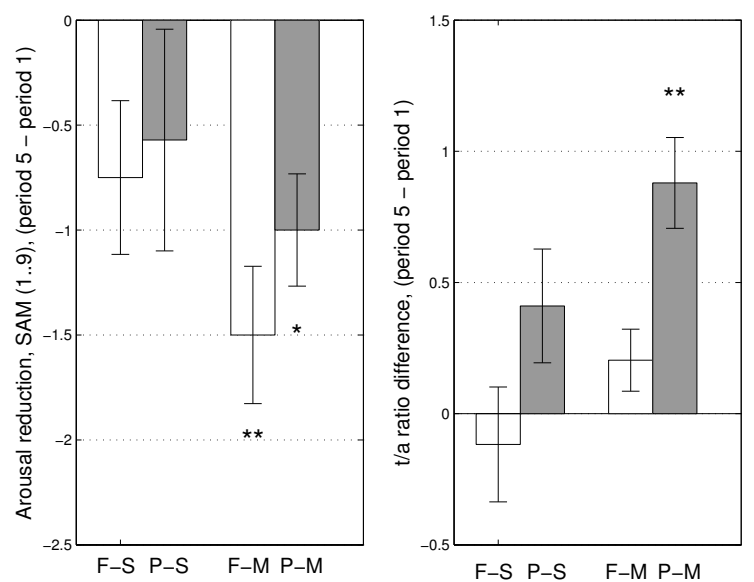

Figure 3: The means of the difference between the first and the final 3-min period of the 15-minute session. Subjective arousal (left panel) and $t / a$ ratios (right panel) are shown for four experimental groups: F-S (Fixed mapping/Single feature), P-S (Personalized mapping/Single feature), F-M (Fixed mapping/Multiple features), P-M (Personalized mapping/Multiple features). Error bars represent standard error values. Bonferroni-corrected significant difference from 0 at $p<0.05(*)$, and at $p<0.01(* *)$ levels.

all MANOVA effect of Relaxation period $\times$ Feedback personalization was significant with $F(5,23)=2.58, p<0.05, \Lambda=0.64, \hat{\eta}_{P}^{2}=0.36$. This effect reached significance both for $t / a$ ratio at $F(1,27)=13.14, p<0.001, \hat{\eta}_{P}^{2}=0.33 ;$ and for relative alpha power, $F(1,27)=10.08, p<$ $0.005, \hat{\eta}_{P}^{2}=0.27$. Changes in relative theta power did not reach significance. For the groups with personalized feedback, $t / a$ ratios increased from $0.73(S E=0.2)$ to $1.42(S E=0.2)$, while for the groups with fixed mappings such change could not be found; the period means $0.93(S E=0.2)$ for the first and $0.79(S E=0.2)$ for the fifth one (see also Figure 3 , right panel). Similar pattern occurred for relative alpha levels, with the means for personalized groups dropping from $0.29(S E=0.04)$ to $0.15(S E=0.03)$, and steady level means for groups with fixed mappings of $0.2(S E=0.04)$. This effect can be also see in Figure 4 (right panel) where differences between the last and the first period are plotted.

In line with our Hypothesis 2, the overall MANOVA effect of Relaxation period $\times$ Number of sonified EEG features was significant with $F(5,23)=$ 5.09, $p<0.005, \Lambda=0.48, \hat{\eta}_{P}^{2}=0.53$. This effect reached significance only for $t / a$ ratio at $F(1,27)=$ $8.94, p<0.01, \hat{\eta}_{P}^{2}=0.25$. Here, the higher number of sonified features in the feedback resulted in a bigger $t / a$ ratio increase from the mean of $0.83(S E=0.2)$ at the initial experiment stage to $1.45(S E=0.2)$ at the final 3-min period. In comparison, groups with single feature based feedback had no improvement, going
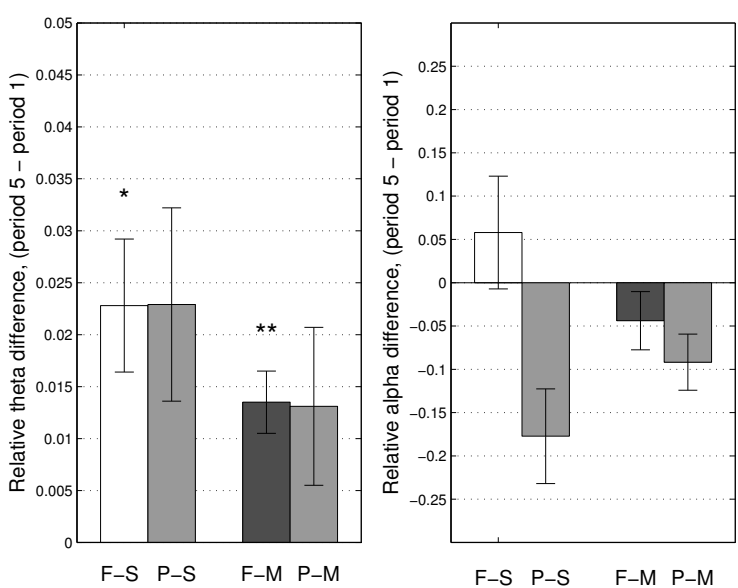

Figure 4: The means of the difference between the first and the final 3-minute period of the 15-minute session. Relative theta power (left panel) and relative alpha power (right panel) are shown for four experimental groups: F-S (Fixed mapping/Single feature), P-S (Personalized mapping/Single feature), F-M (Fixed mapping/Multiple features), P-M (Personalized mapping/Multiple features). Error bars represent standard error values. Bonferroni-corrected significant difference from 0 at $p<0.05(*)$, and at $p<0.01(* *)$ levels.

from the mean of $0.84(S E=0.2)$ to $0.76(S E=0.2)$, see also Figure 3 (right panel).

The triple interaction between Relaxation period $\times$ Feedback personalization $\times$ Number of sonified features could be also observed. It reached significance only for the relative alpha, $F(1,27)=5.13, p<$ $0.05, \hat{\eta}_{P}^{2}=0.16$. This effect can be better seen in Figure 4 (right panel), where differences between two periods are plotted for each of participants' group. While not significant when comparing the period differences within each group, this interaction is reflected in bigger difference between F-S and P-S groups as compared to the difference between F-M and $\mathrm{P}-\mathrm{M}$ groups.

\section{DISCUSSION}

The results from our between-group validation of sonifications using a/t neurofeedback training confirmed the two initial hypotheses, both for subjective and objective data. The significant differences between initial and final relaxation periods for P-S and $\mathrm{P}-\mathrm{M}$ groups using personalized feedback support our first hypothesis that personalized EEG-to-Sound mappings will be more instrumental than the fixed ones. A significant increase in $t / a$ ratio and decrease in alpha relative power were observed after 15-min NF session. This trend was not repeated for F-S and F-M user groups training with fixed sonification mappings.

Our second hypothesis, stating that multiple EEG 
feature sonifications will be more efficient than those relying on a single EEG feature, was also confirmed. A significant increase of $t / a$ ratio and a significantly lower reported subjective arousal after the NF session was observed only for P-M and F-M groups with multiple feature feedback. As expected, the training effect was smaller for P-S and F-S groups training with single feature mappings.

Finally, changes in relative alpha power showed an interaction between a number of sonified EEG features and their personalization. Here, the differences between the P-S and F-S groups that were using fixed and personalized sonifications of a single feature are bigger than between P-M and F-M groups whose training was based on multiple EEG features. In other words, personalization became less instrumental when multiple features were sonified. Here, the decrease in attentional resources could explain this effect. As a number of sound objects to attend becomes larger, it is more likely that "change deafness" might reduce sonification efficiency (see a recent review by (Shinn-Cunningham, 2008) on auditory spatial attention). Certainly, the complexity of auditory feedback is an object of a separate study.

Significant between-group differences in subjective and physiological data show that the observed results are not obtained merely due to relaxing nature of presented soundscapes (wind, water, etc.). While we did not aim to study specifically the neurofeedback effects, the full procedure of a/t NF training with realtime feedback differed from simple relaxation. Undeniably, more training sessions, larger number of participants, and more robust EEG equipment should be used to study brain dynamics during a/t training (e.g. changes between training sessions, learning curves within each NF session, individual differences etc.). However, the observed results that varied significantly between four test groups support the full functionality of the conducted NF training. Therefore, we can argue that both sonification richness (a number of EEG features to be displayed) and end-user personalization play an important role in the effectiveness of real-time EEG sonification for neurofeedback.

Our study did not include Sham group with fake neurofeedback or Control group doing 15-minunte relaxation to compare with our results. While such control would be necessary to quantify the depth of relaxation with and without neurofeedback, this question was not in scope of our study. As predicted, and also as shown by the pre- and post-training differences, the participants group F-S with fixed sonification of the single EEG feature can be seen as a strongest baseline for the three other groups.

Several observations can be made when compar- ing our results with previous works on sonification richness and personalization. The transparency of the controls for sound adjustment was an important factor, as in the case of sonification-based EEG diagnostic by expert users (De Campo et al., 2007). The positive effect of personalization in our studies demonstrates that the task of sonification adjustment was clear and understandable for naive end-users. However, generation and direct evaluation of intuitive interfaces for both end-users and experts should be further explored. In this regard, we plan to integrate our sonification system into a tabletop, tangible interface. Our previous work shows that such multimodal Brain-Tangible User Interfaces (BTUI) foster performance and motivational aspects of interaction, allowing for multi-user setups with shared interface control for training and collaborative work (Mealla et al., 2011).

Another issue is the problem of validation in sonification studies as pointed out by (Väljamäe et al., 2013). While diagnostic and neurofeedback applications are expected to have a rigorous assessment, most of EEG sonification studies lack a proper validation, making difficult to determine the efficiency of a particular EEG sonification strategy. Certainly, this lack of quantitative assessment reflects the ongoing development in this research field. In this context, we contributed to the methodologies for sonification validation through a combination of objective and subjective measures using a well-known neurofeedback scenario. Some discrepancy between subjective ratings and physiological indices observed in our study should encourage the use of multimodal measures.

Finally, it is important to note a number of advantages offer by the presented sonification system. First, model-based sonification provides a wide range of richness and manipulation, versatile EEG-to-Sound mapping, and realistic dynamic sounds. Here, several EEG features can be used as an input to one model, e.g. rain model, where its several parameters can vary (amount, loudness, sound characteristics). Second, the use of Pd permits a modular approach, allowing the combination of different sound objects and the addition of new ones. Special interest here present sounds that are directly related to user emotional state like breathing or heart beat (Tajadura-Jimenez et al., 2008). Other future work include testing different NF protocols, integrating and evaluating the system with other EEG devices (Mealla et al., 2011), and studying user preferences for sound mappings and their effectiveness for medical or training applications. Finally, future work regarding the $a / t$ training protocol will imply tests with several sessions, different sound models and other EEG equipment. 


\section{CONCLUSIONS}

A real-time sonification system was used to design flexible, multi-parametric EEG data sonifications, that were later adjusted in real time by endusers to personalize the mappings. Four settings varying in feedback richness and personalization were evaluated using 15-min a/t neurofeedback training and between-subjects design. Comparing both subjective and physiological data from pre- and posttraining showed significant relaxation in groups with personalized feedback, while not in groups training with fixed sonification mappings. In addition, a larger number of sonified EEG features resulted in higher $t / a$ ratio increase. These results demonstrate that both sonification richness (a number of EEG features to be displayed) and end-user personalization play an important role in the effectiveness of real-time EEG sonification for neurofeedback.

\section{ACKNOWLEDGEMENTS}

The last author of this paper received funding from Marie Curie Actions of the European Union's Seventh Framework Programme (FP7/2007-2013) under REA GA-303172.

\section{REFERENCES}

Allanson, J. and Fairclough, S. (2004). A research agenda for physiological computing. Interacting with Computers, 16(5):857-878.

Bradley, M. M. and Lang, P. J. (1994). Measuring emotion: The self-assessment manikin and the semantic differential. Journal of Behavior Therapy and Experimental Psychiatry, 25(1):49 - 59.

De Campo, A., Hoeldrich, R., Eckel, G., and Wallisch, A. (2007). New Sonification Tools For Eeg Data Screening And Monitoring. In Proceedings of the 13th International Conference on Auditory Display, volume 67(2009)90, pages 536-542.

Duvinage, M., Castermans, T., Petieau, M., Hoellinger, T., Cheron, G., and Dutoit, T. (2013). Performance of the emotiv epoc headset for p300-based applications. Biomed Eng Online, 12:56.

Egner, T., Strawson, E., and Gruzelier, J. (2002). Eeg signature and phenomenology of alpha/theta neurofeedback training versus mock feedback. Applied Psychophysiology and Biofeedback, 27(4):261-270.

Emotiv (2013). Emotiv epoc. http://www.emotiv.com.

Gruzelier, J. (2009). A theory of alpha/theta neurofeedback, creative performance enhancement, long distance functional connectivity and psychological integration. Cognitive Processing, 10(1):101-109.
Guttman, S. E., Gilroy, L. A., and Blake, R. (2005). Hearing what the eyes see: Auditory encoding of visual temporal sequences. Psychological Science, 16(3):228-235.

Hermann, T., Meinicke, P., and Bekel, H. (2002). Sonifications for EEG data analysis. In Proceedings of the International Conference on Auditory Display (ICAD 2002), pages 3-7, Kyoto.

Hjorth, B. (1970). Eeg analysis based on time domain properties. Electroencephalography and clinical neurophysiology, 29(3):306-310.

Khamis, H., Mohamed, A., Simpson, S., and McEwan, A. (2012). Detection of temporal lobe seizures and identification of lateralisation from audified EEG. Clinical Neurophysiology, 123(9):1714-20.

Kropotov, J. (2010). Quantitative EEG, event-related potentials and neurotherapy. Elsevier.

Liang, S.-F., Chen, Y.-C., Wang, Y.-L., Chen, P.-T., Yang, C.-H., and Chiueh, H. (2013). A hierarchical approach for online temporal lobe seizure detection in long-term intracranial eeg recordings. Journal of neural engineering, 10(4):045004.

Mealla, S., Väljamäe, A., Bosi, M., and Jordà, S. (2011). Listening to your brain: Implicit interaction in collaborative music performances. In Proc. of NIME, pages 149-154.

Mullen, T., Luther, M., Way, K., and Jansch, A. (2011). Minding the (Transatlantic) Gap: An Internet-Enabled Acoustic Brain-Computer Music Interface followed throughout the next decade by a number of artists. In Proc. NIME'11.

Neuroelectrics (2013). Neuroelectrics enobio. http://neuroelectrics.com/enobio.

Puckette, M. et al. (1996). Pure data: another integrated computer music environment. Proceedings of the Second Intercollege Computer Music Concerts, pages 37 41.

Rodríguez, A., Rey, B., and Alcañiz, M. (2013). Validation of a low-cost eeg device for mood induction studies. Studies in health technology and informatics, 191:4347.

Shinn-Cunningham, B. G. (2008). Object-based auditory and visual attention. Trends Cogn Sci, 12(5):182-186.

Tajadura-Jimenez, A., Valjamae, A., and Vastfjall, D. (2008). Self-representation in mediated environments: the experience of emotions modulated by auditoryvibrotactile heartbeat. Cyberpsychology and Behavior, 11(1):33-38

Väljamäe, A., Mealla, S., Steffert, T., Holland, S., Marimon, X., Benitez, R., and et al. (2013). A Review Of Real-time EEG Sonification Research. In The 19th International Conference on Auditory Display (ICAD. 2013), Lodz, Poland.

Vastfjall, D. (2003). The subjective sense of presence, emotion recognition, and experienced emotions in auditory virtual environments. Cyberpsycholy and Behavior, 6(2):181-188.

Wright, M. (2005). Open sound control: an enabling technology for musical networking. Organised Sound, 10(03):193-200. 